\title{
Various Complications of Chickenpox in Admitted Patients; 2017 Perspective
}

\author{
Aamir Shaukat ${ }^{1}$, Umair Ahmed ${ }^{2}$, Hooria Aamir ${ }^{3}$, Uzma Ismail ${ }^{4}$, Mammona Jahangir $^{5}$, Awais Ahmad ${ }^{6}$
}

Professor, Department of Medicine, Faisalabad Medical University, Faisalabad Pakistan

1 Title Selection, Concept of Research

2 Assistant Professor, Department of Medicine, Faisalabad Medical University, Faisalabad Pakistan

Writing Manuscript, Compiling Data

3 Associate Professor, Department of Physiology, Faisalabad Medical University, Faisalabad Pakistan References Writing, Compiling Data

Medical Officer, Allied Hospital, Faisalabad Pakistan

4 Manuscript Writing, Data Collection

5 Medical Officer, Allied Hospital, Faisalabad Pakistan

Data Collection, Compiling Data

Senior Registrar, Department of Radiology, Allied Hospital, Faisalabad Pakistan

References Writing, Proof Reading

\author{
Submitted for Publication: 05-01-202 \\ Accepted for Publication 28-09-2020 \\ CORRESPONDING AUTHOR \\ Prof. Dr. Aamir Shaukat \\ Professor, Department of Medicine, \\ Faisalabad Medical University, Faisalabad Pakistan \\ Email:dr_aamirfcps@yahoo.com
}

\section{ABSTRACT}

Background: Chickenpox is a benign disease occurring in both children and adults. Common symptoms of disease include fever, generalized body rash, itching, fatigue, headache and body aches. The complications of the disease include respiratory, gastrointestinal, neurological, hematological and cardiac complications. These complications are more common in adult population. Estimated mortality from chickenpox is 31/100,000 in adults. Chickenpox pneumonia is the most serious complication followed by encephalitis. Objective: To highlight the various complications of chickenpox present in adult population of Faisalabad and its adjoining cities. Study Design: retrospective cross-sectional observational study. Settings: Department of Medicine, Allied Hospital, Faisalabad Pakistan. Duration: One year, January 2017 to December 2017. Methodology: Record of the admitted 350 patients was obtained, and Patients' charts were reviewed with special emphasis on various complications of chickenpox. The patients included were above the age of 14 years and the diagnosis of chickenpox was made on clinical grounds. The diagnosis of complications was made with the help of laboratory and radiological investigations. Results: In our study 350 patients facing complications from chickenpox, from various regions of Faisalabad. Majority of them suffered from secondary bacterial skin infection i.e. 75 patients. Sepsis and ARDS turned out to be some of the major concerns for patients as well, as 31patients developed Sepsis and 29 patients suffered from ARDS respectively. Hepatitis, Myocarditis and Encephalitis were among the major issues affecting 11, 12 and 9 patients respectively. Conclusion: Chicken Pox can present with life threatening complications in adult population. Awareness of general population about the disease, its prevention, early detection and start of appropriate treatment can prevent development of complications and help reduce mortality and morbidity from the disease.

Keywords: Encephalitis, Pneumonia, Septicemia, Varicella zoster.

How to cite: Shaukat A, Ahmed U, Aamir H, Ismail U, Jahangir M, Ahmad A. Various Complications of Chickenpox in Admitted Patients; 2017 Perspective. APMC 2020;14(4):292-4. DOI: 10.29054/APMC/2020.1005

\section{INTRODUCTION}

Chickenpox is a benign disease occurring in both children and adults. Although it has a high frequency among pediatric population, cases of chickenpox are also reported widely among adult population. The reason for decreasing cases among younger population is unclear but this had led to non-immunized adult population who is more prone to develop complications of chicken pox. ${ }^{1}$ Usual incubation period of chickenpox is 14-16 days. Common symptoms of disease include fever, generalized body rash, itching, fatigue, headache and body aches. The usual benign course of the disease has actually overshadowed the complications of the disease which include secondary infections, respiratory, gastrointestinal, neurological, hematological and cardiac complications to state the a few. ${ }^{2}$

Secondary infections including skin and respiratory tract are the most common complication of chickenpox. Chickenpox pneumonia is the most serious complication with high mortality. Other complications with high frequency are the neurological. This group includes encephalitis, meningoencephalitis, Guillain-Barre syndrome, cerebellar ataxias, transverse myelitis among others. Gastrointestinal complications include hepatitis, gastritis and rarely pancreatitis. Myocarditis and pericarditis ${ }^{3}$ are also reported. Secondary re-activation of chickenpox can lead to herpes zoster and its complications as well. 
Estimated mortality from chickenpox is $31 / 100,000$ in adults. The most common cause of mortality include pneumonia leading to Adult Respiratory distress Syndrome (ARDS) and encephalitis. It has been estimated that mortality from ARDS might be as high as 30\% and this number increases to $50 \%$ in patients who are put on mechanical ventilation.

The rate of these complications and associated morbidity and mortality might be decreased with the availability of varicella zoster vaccination. It is available freely in market, but it has not yet been introduced as part of the vaccination programs. There are still questions about the effectiveness of vaccination to control complications in adults as long-term studies are not yet available. Another hindrance to the use of vaccination is its costeffectiveness. The purpose of our study is to highlight the various complications of chickenpox present in adult population of Faisalabad and its adjoining cities.

\section{METHODOLOGY}

Study Design: Retrospective cross-sectional observational. Settings: Department of Medicine, Allied Hospital, Faisalabad Pakistan.

Duration: One year from January 2017 to December 2017. Sample Technique: Non-probability consecutive sampling technique was used.

Sample Size: 350 patients were studied.

Inclusion Criteria: The patients included were above the age of 14 years and the diagnosis of chickenpox was made on clinical grounds.

Exclusion Criteria: The patients who did not give consent were excluded from the study.

Data Collection Procedure: Record of the admitted patients was obtained, and Patients' charts were reviewed with special emphasis on various complications of chickenpox. The diagnosis of complications was made with the help of laboratory and radiological investigations.

\section{RESULTS}

There were total 350 patients facing complications from chickenpox, from various regions of Faisalabad. Several major and minor complications were encountered by patients, however, majority of them suffered from secondary bacterial skin infection i.e. 75 patients, out of which 51 were male and 24 of them were females. But sepsis and ARDS turned out to be some of the major concerns for patients as well, as 31 patients developed sepsis and 29 patients suffered from ARDS respectively. Nonetheless, hepatitis, myocarditis and encephalitis were among the major issues faced by 11, 12 and 9 patients respectively. Furthermore, there were some of the minor complications like pneumonia without RDS, pancreatitis, DIC, GBS, transverse myelitis and gastroenteritis that affected the patients.
Table 1: Frequency of complications in patients with chicken pox

\begin{tabular}{|l|c|}
\hline Complications & $\begin{array}{c}\text { No. of patients } \\
\text { suffered }\end{array}$ \\
\hline Secondary bacterial skin infection & 75 \\
\hline ARDS & 29 \\
\hline Pneumonia without ARDS & 4 \\
\hline Hepatitis & 11 \\
\hline Pancreatitis & 2 \\
\hline Myocarditis & 12 \\
\hline Sepsis and septic shock & 31 \\
\hline DIC & 3 \\
\hline Encephalitis & 9 \\
\hline GBS & 2 \\
\hline Transverse myelitis & 1 \\
\hline Gastroenteritis & 2 \\
\hline
\end{tabular}

\section{DISCUSSION}

Chickenpox might manifest itself aggressively in adults. It can express itself in the form of immunosuppression, respiratory involvement or neurological involvement, which results in increased mortality rate. Dermatological complications too manifest because of chickenpox.

VZV pneumonia is one of the most serious complications of this infection in adults. Smoking has been proven to be a major risk factor for a severe disease in varicella pneumonia by various studies in the past. One of the studies published by Ellis et al successfully proved that smoking was a risk factor for development of severe

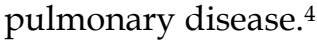

Currently, VZV-induced pneumonia is considered to be one of the most serious complications of VZV infection.

Cerebellar ataxia is the most common neurological complication of chickenpox. It accounts for more than one half of all the neurological complications of varicellazoster virus (VZV) infection. ${ }^{5}$ It is characterized by ataxia with nystagmus, headache, nausea, vomiting and nuchal rigidity. ${ }^{5}$ The symptoms may begin as early as 11 days before and up to 21 days after the onset of the rash. ${ }^{5}$ The examination of CSF in varicella-associated cerebellar ataxia reveals nonspecific findings with a cell count ranging from 0 to 68 cells $/ \mathrm{m}$. Lymphocytic pleocytosis and elevated protein may occur in $20-30 \%$ cases. The CSF glucose is normal and polymorphonuclear cells may be present in the early course of the disease..$^{5}$ In mild cases, no further evaluation is required. ${ }^{6}$ Neurological complications can antedate cutaneous eruptions, particularly in men. Detection of viral antigens by polymerase chain reaction or advanced fragment analysis can be helpful to diagnose such presentation.7,8 Chickenpox encephalitis is too reported among adults and infants. It represents itself as hypertonia or hypotonia, hemiparesis, positive planter responses etc. Post herpetic neuralgia is another complication found among affected individuals. Other CNS manifestations 
including disturbed level of consciousness, coma, seizures, motor deficits, ataxia, and myoclonus, and the most common finding on CT scan was brain edema. ${ }^{9}$

Various lesions present on the skin and soft tissue lead to different skin complications following varicella infections such as idiopathic purpura fulminans and varicella gangrenosa of the both hands, toes, and integument. The skin and soft tissue lesions can also lead to complications like nacrotizing fasciitis, streptococcal toxic shock syndrome and secondary bacterimeia. ${ }^{1}$

\section{CONCLUSION}

Chicken Pox can present with life threatening complications in adult population.

Awareness of general population about the disease, its prevention, early detection and start of appropriate treatment can prevent development of complications and help reduce mortality and morbidity from the disease. Mass Campaigns regarding the Vaccination of Chicken Pox can go a long way in reducing the burden of this disease especially in adult population.

\section{LIMITATIONS}

The study was conducted in a single center. Large scale studies involving multiple centers would give a clear picture about the complication of chicken pox in adult population.

\section{SUGGESTIONS / RECOMMENDATIONS}

Vaccination for prevention of chickenpox should be made mandatory in EPI program of immunization. So as to prevent adults and children from developing chickenpox.

\section{CONFLICT OF INTEREST / DISCLOSURE}

No.

\section{ACKNOWLEDGEMENTS}

The author acknowledged the efforts of front-line doctors, paramedical staff who perform duties in isolation ward taking care of patients with chickenpox.

\section{REFERENCES}

1. Hasan T, khader K. Significance of adult chicken pox infections in Saudi Arabia:, A case report. Anatomy Physiol Biochem Int J. 2017; 4(1): 555629

2. Ayyad A, Rkain M, Babakhouya A, Benajiba N. Varicella is not always benign. Pan Afr Med J. 2018;31:30.

3. Dogan OA, Topcu S, Tanir NG. Varicella-related hospitalizations among immunocompetent and immunocompromised children in pre-vaccine era: a tertriary care center experience in Turkey. J Pediatr Res. 2018; 5(1):11-6.

4. Ellis ME, Neal KR, Webb AK. Is smoking a risk factor for pneumonia in adults with chickenpox?. Br Med J (Clin Res Ed). 1987;294(6578):1002-8.

5. Varela FH, Pinto LA, Scotta MC. Global impact of varicella vaccination programs. Hum Vaccin. 2019;15(3):645-57.

6. Scheld WM, Whitley RJ, Durack DT. Infections of the central nervous system. Raven Press: New York, NY; 1991:59.

7. Song Y, Li Ye, Jiang Y, Liu T. Detection of varicella-zoster virus from cerebrospinal fluid using advanced fragment analysis in a child with encephalitis: a case report. BMC Infect Dis. 2019;19:342.

8. Puchammar Stockl E, Popow-Kraupp T, Heinz FX, Mandi CW, Kanz C. Detection of zoster virus DNA by polymerase chain reaction in the cerebrospinal fluid of patients suffering from neurological complications associated with chickenpox or herpes zoster. J Clin Microbiol. 1991;29:1513.

9. Abdul Hafiz KM, Abdul Moneim AA, Betar Z, Meraj MA, Abdul Kalik Y. The immunological aspects of acute C.N.S. complications of the exanthematous viral diseases. J Egypt Soc Parasitol. 1996;26(1):169-76. 